\title{
Development of a Matrix-prespotted Plate for Enhancing the Reproducibility of Serum Glycan Analysis by MALDI-TOF-MS
}

\author{
Miyoung Ha ${ }^{\text {a,b }}$, Youngha In ${ }^{\mathrm{a}}$, Hyesun Maeng ${ }^{\mathrm{a}}$, Ok Pyo Zee ${ }^{\mathrm{b}}$, Jongsik Lee ${ }^{\mathrm{a}}$, and Yangsun Kim ${ }^{\mathrm{a}, *}$ \\ ${ }^{a}$ Applied Surface Technology Inc., 864-1 Ieudong, Suwon 443-270, Korea \\ ${ }^{b}$ College of Pharmacy, Sungkyunkwan University, Suwon 440-746, Korea
}

Received September 9, 2011; Revised Septermber 14, 2011 ; Accepted September 14, 2011

First published on the web September 15, 2011; http://dx.doi.org/10.5478/MSL.2011.2.3.061

\begin{abstract}
Matrix Assisted Laser Desorption/Ionization-Time-of-Flight mass spectrometry (MALDI-TOF-MS) is the most widely used MS technique for glycan analysis. However, the poor point-to-point and sample-to-sample reproducibility becomes a limit in glycan biomarker research. A prespotted MALDI plate which overcomes the large crystal formation of 2,5-dihydroxybenzoic acid (DHB) has been developed and applied for glycan analysis. A homogeneous matrix coated surface without a crystal structure was formed on a hydrophilic/ hydrophobic patterned surface using a piezoelectric device. The reproducible MALDI-TOF-MS data have been presented using MALDI imaging of beer glycan as well as serum glycan eluted from $10 \%$ and $20 \%$ ACN elution fractions. The glycan profile from the serum glycan by MALDI-TOF-MS with a DHB prespotted plate was highly conserved for 10 different spectra and the coefficient of variations of significant ion peaks of MALDI data varies from 3.59 to 19.95.
\end{abstract}

Key words: MALDI-TOF-MS, Glycan analysis, Prespotted MALDI plate

\section{Introduction}

Glycosylation is one of most common forms of cotranslational and post-translational modifications ${ }^{1}$ (Wikipedia, term "glycosylation"). Glycans serve variety of structural and functional role in a cell membrane and secreted proteins such as cell to cell recognition process, infection, and biological markers for a wide variety of diseases., ${ }^{2,3}$ Therefore, analysis of glycan profiles or glycosylation pattern of glycoproteins is important and their comparison of it in normal and disease states is critical in biomarker research. ${ }^{4}$

Matrix Assisted Laser Desorption/Ionization (MALDI) is one of the most widely used MS techniques for glycan analysis ${ }^{5,6}$ since it provides accurate information of glycan structure and linkage. 2,5-dihydroxybenzoic acid (DHB) is a widely accepted matrix for most glycan analysis in MALDI MS, and different approaches and matrix preparation are needed for the analysis of neutral and acidic glycans. ${ }^{7,8}$ Neutral glycans are ionized well in the positive ion mode using DHB and their ionization efficiency increases with an alkaline ion additive. Acidic glycans show variable losses of sialic acids in the positive mode, and therefore were analyzed in the negative ion mode with DHB and ammonium citrate as additives. ${ }^{9}$

Even though 2,5-DHB matrix is sufficient for most glycan profile applications, the notorious nature of sample preparation in MALDI itself such as a presence of a "sweet

*Reprint requests to Dr. Yangsun Kim

E-mail: yangskim@maldiplate.com spot" which comes from heterogeneous crystal formation of sample and matrix, prevents from obtaining reproducible results. $^{10-12}$ Since MALDI-TOF MS has an advantage of high throughput capability compared with other MS techniques and comparison of a large set of data from normal and patient samples is essential for biomarker research, enhancing the reproducibility of MALDI data will contribute to a progress in the field.

In this work, to avoid point to point and spot to spot variation of signal intensity due to the large crystal formation of DHB matrix, a DHB prespotted MALDI plate using a piezoelectric spotting system has been developed and applied for glycan analysis. The homogeneous matrix spots ${ }^{13}$ and their change of matrix crystal structures during sample preparation have been monitored and reproducible MS results have been demonstrated with beer glycans as well as $\mathrm{N}$ - glycans from serum.

\section{Experimental}

\section{Materials and samples}

The 2,5-dihydroxybenzoic acid (2,5-DHB, >99.0\%), trifluoroacetic acid (TFA), sodium chloride, hydrochloride, were purchased from Sigma-Aldrich (St. Louis, MO, USA). HPLC grade organic solvents, acetonitrile (ACN), and ethanol were purchased from Merck (Darmstadt, Germany). Beer glycan sample was prepared from commercially available beer and aged for $24 \mathrm{hrs}$ at $4^{\circ} \mathrm{C}$ for degassing. Human serum samples were purchased from Sigma Aldrich (lot number Sigma-H4522) and glycerol free endo glycosidase F (peptide: $\mathrm{N}$-glycosidase F, PNGase F) was purchased from New 
England Biolabs (Ipswich, USA).

\section{Fabrication of matrix-patterned surface}

The hydrophilic and hydrophobic patterned templates with $1700 \mu \mathrm{m}$ spots were developed by chemical etching and photolithography used in semiconductor production. Matrices and chemicals for surface modification have been deposited on hydrophilic spots in patterned templates by an optimized piezoelectric device with $5760 \times 1440$ dpi resolution and a six channel piezoelectric head that delivers 87 pL per $200 \mu \mathrm{m}$ square. The microscopic image of spot is in Figure 1. The matrix crystals have diameters of about a few tens of micrometers, spaced around $20 \mu \mathrm{m}$ apart. ${ }^{13} 100 \mathrm{mg} / \mathrm{ml} 2,5-\mathrm{DHB}$ in ACN/ $0.1 \%$ TFA solution $(7: 3, \mathrm{v} / \mathrm{v})$ mixed with $10 \mathrm{mM}$ sodium chloride as a cation additive was used. The CorelDRAW software was used to create an image template corresponding to the sample location.

\section{Sample preparation}

Beer glycan was obtained by dilution of beer in ACN/water $(1: 1, \mathrm{v} / \mathrm{v})$ at a concentration of $10 \%$. Serum glycan was prepared following the protocol in ref. $14^{14}$ as shown in scheme 1. For example, $50 \mu \mathrm{l}$ serum was denatured with DTT, deglycosylated with 1 unit of PNGase $\mathrm{F}$ at $37^{\circ} \mathrm{C}$ for 30 min with a microwave ultrafast digestion system at 400 watt power. Ethanol precipitation treatment with $80 \%$ cold ethanol was applied to remove the serum proteins. The obtained oligosaccharides were purified by Solid Phase Extraction using a graphitized carbon cartridge (GCC) (Alltech, Deerfield, IL). The cartridges were washed with nanopure water followed by $0.05 \%(\mathrm{v} / \mathrm{v})$ TFA in $80 \% \mathrm{ACN}$ in $\mathrm{H}_{2} \mathrm{O}(\mathrm{v} / \mathrm{v})$ and finished up with nanopure water again before loading the serum glycan solution. $10 \%$ ACN in $\mathrm{H}_{2} \mathrm{O}(\mathrm{v} / \mathrm{v})$ and $20 \% \mathrm{ACN}$ in $\mathrm{H}_{2} \mathrm{O}$ were applied sequentially to obtain purified $\mathrm{N}$-glycans from the serum. These fractions cover most of neutral N-glycans from serum while $40 \%$ fraction includes acidic glycans which could be

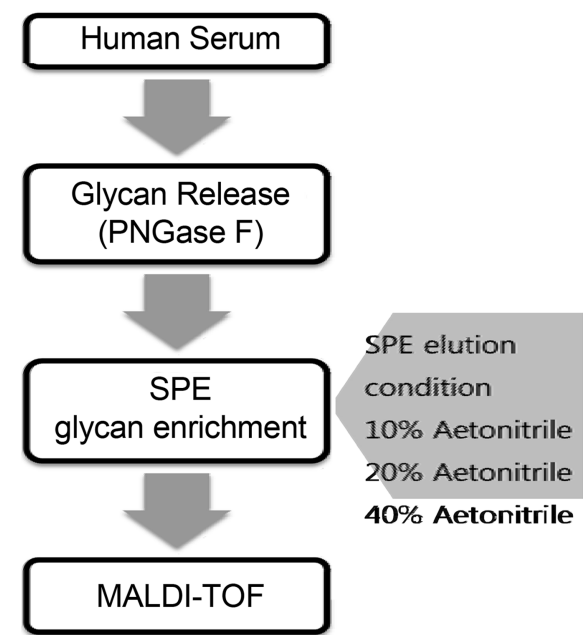

Scheme 1. The procedure for preparation of serum glycan for MALDI-TOF analysis.
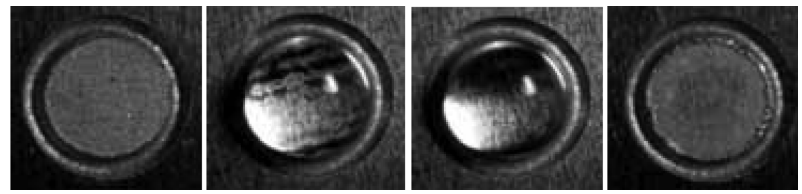

Figure 1. Microscopic image of a DHB prespotted spot and sample preparation on it.

identified with negative MALDI. The obtained sample was concentrated and applied directly on a matrix prespotted MALDI plate for MALDI-TOF MS analysis. When the sample is loaded on prespotted plates, the change of the spot was shown in Figure 1. Even after loading a sample and dried in vacuum, the homogeneity of sample spot was preserved. Careful sample spot preparation with uniform microcrystal of analytes/matrix promotes a more uniform distribution of the analyte throughout the spot and improves point-to-point and sample-to-sample reproducibility.

\section{Mass Spectrometry}

A Ultraflex MALDI-TOF mass spectrometer (Bruker Daltonics, Bremen, Germany) equipped with the smart beam was operated with reflectron, and positive ion mode for MALDI imaging of beer glycan analysis. Data preparations such as average peak list calculation, peak calculation, recalibration, and peak statistic results were performed by ClinproTool 2.1 software (Bruker Daltonics). For serum glycan, AB Sciex 4800 MALDI- TOF-TOF system has been used in the positive ion mode with reflectron.

\section{Results \& Discussion}

MALDI-TOF MS is an attractive technique for reasons of its sensitivity, reliability, and high throughput capability. ${ }^{15}$ However, it is not considered a suitable quantitative technique because of non-uniform distribution of analytes during matrix co-crystallization. ${ }^{10} 2,5$-DHB is a preferred matrix for glycan analysis, but due to the large crystal formation during the common dried-droplet sample preparation, point-to-point signal reproducibility is the worst among other matrices such as 4-hydroxy-3-methoxycinnamic acid (ferulic aicd, FA); sinapinic acid (SA); 3,4-dhydroxycinnamic acid (CA); 3-hydroxypicolinic acid (3-HPA); 2-(4-hydroxyphenylazo) benzoic acid (HABA); $\alpha$-cyano-4-hydroxycinnamic acid (CHCA). ${ }^{16}$

Since glycan profiles from human serum reflect some of cancers and their states, comparison of human serum glycan profiles between the normal and cancer state is important in a cancer biomarker research. ${ }^{17}$ Therefore, if a correlation between the signal intensity of MALDI-TOF MS and glycan abundance has been set up, high throughput screening of glycan biomarkers become feasible. In this regards, reproducible data acquisition from MALDI TOF could be the first step to be achieved. Figure 1 shows the microscopic image of a DHB prespotted spots. After loading $1 \mu \mathrm{l}$ of glycan sample and vacuum dried, the initial homogeneous surface conditions 
are conserved and the notoriously long crystal structure of DHB was not observed. MALDI MS spectrum of beer glycan is in Figure 2(A) and the spot to spot variation is shown in Figure 2(B) with a MALDI image for a dominating peak, $1337.6 \mathrm{~m} / \mathrm{z}$ in all spots. From the image in Figure 2(B), the reproducible ion spectrum could be obtained by automatic picking up sampling area in the center part. The representative spectrum of serum N-glycan of 10\% SPE elution and 20\% SPE elution were shown in Figure 3(A) and 3(B) respectively. The base peaks and the total glycan profile of the spectra were corresponded to those of FTMS presented by An et $a l^{14}$. Most abundant glycan peaks are correspond with those from FTMS data and tentative confirmation of the peaks as glycans

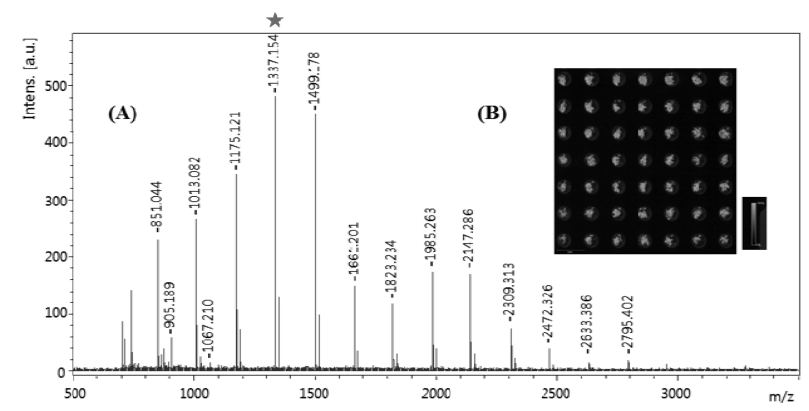

Figure 2. (A) MALDI MS spectrum and (B) MALDI imaging of beer glycan from each spots.

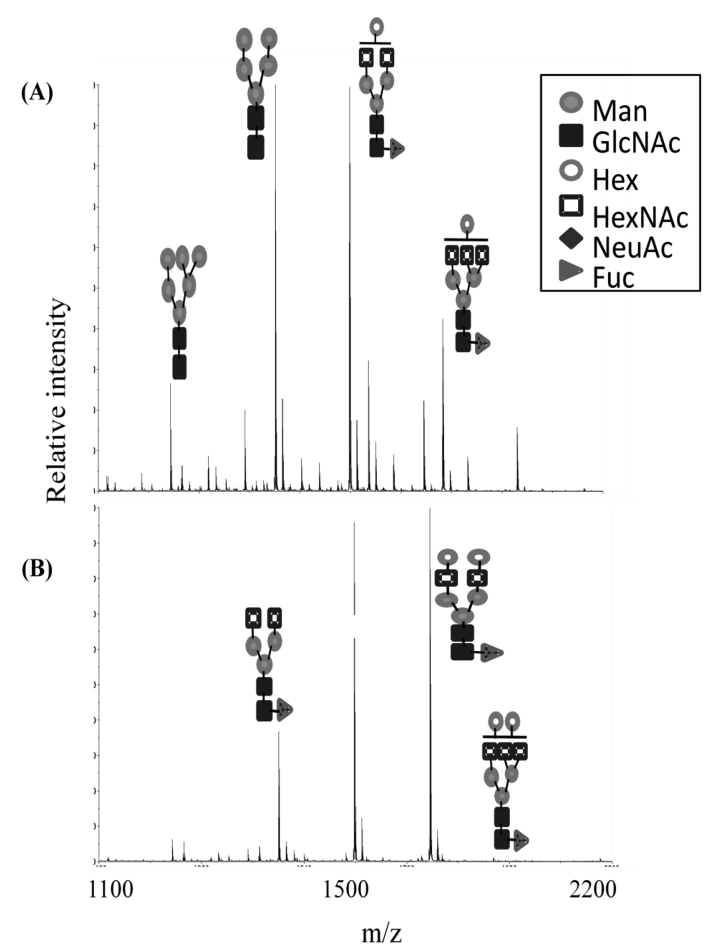

Figure 3. MALDI spectra of serum glycans (A) $10 \% \mathrm{ACN}$ elution fraction and (B) $20 \%$ ACN elution fraction. Annotated putative structure are described based on common serum glycobiology was assigned followed by them. ${ }^{14}$ To compare point-to-point and spot-to-spot reproducibility for serum glycans, 10 different spots were analyzed respectively and the mass spectra were acquired automatically with 200 laser shots from different locations in each spot. The representative spectra from each spot is shown in Figure 4 for $10 \%$ ACN elution and similar conservations was found for $20 \% \mathrm{ACN}$ elution. The even response of glycan profile across all spectra demonstrates the homogeneity of the entire MALDI spots and reproducibility of the ionization process. Reproducibility of the spectra was confirmed with the reconstruction of normalized intensity of significant peaks from spectra for $10 \% \mathrm{ACN}$ elution as well as $20 \%$ ACN elution from Figure 4 . The coefficient of variations $(\mathrm{CV}$, standard deviation/mean) were calculated for those significant peaks. All spectra were normalized by a base peak and shown in Figure 5(A) and Figure 5(B) and the relative intensity, standard deviation and CVs are in Table 1. The standard deviation for relative intensity is less than $10 \%$ for high abundant glycan and $20 \%$ for lower abundant glycan. MALDI-TOF MS is known to have 10 to $20 \%$ distribution in signal intensity coming from instrumental factors. Therefore, the intensity variation by using the DHB prespotted plates is within the instrumental factor. Commercially available matrix prespotted plates with CHCA have been found to give more reliable identification of peptides. ${ }^{18}$ A high density MALDI plate with DHB was successfully applied for high throughput small molecule screening. ${ }^{19}$ The reproducible glycan spectra with DHB prespotted plates could be the base of high throughput screening in glycan biomarker researches especially for high abundant glycan.

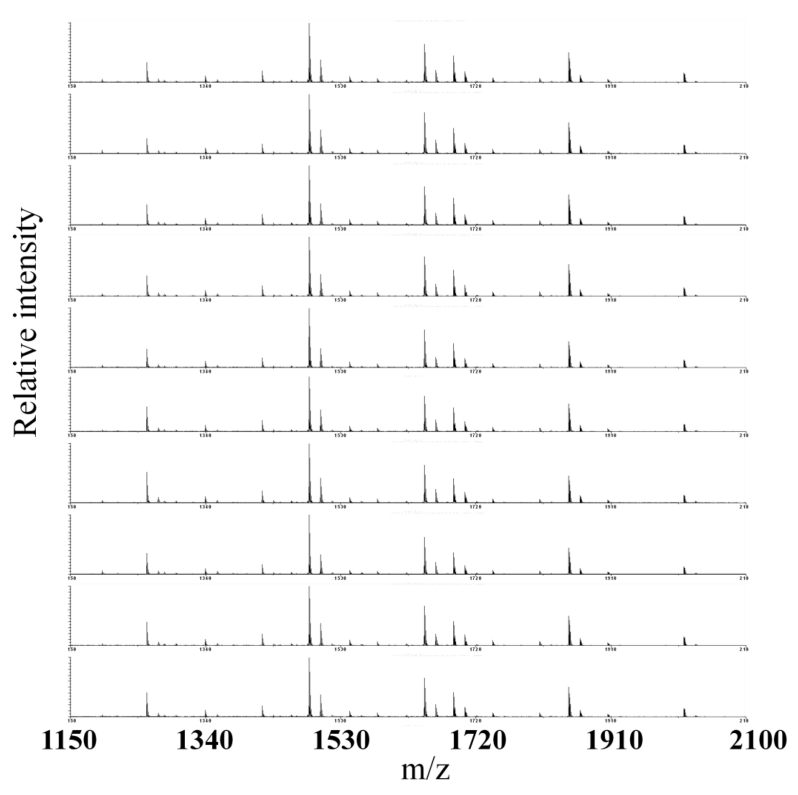

Figure 4. A set of 10 spectra of the $10 \% \mathrm{ACN}$ elution fraction of treated serum glycan from 10 different DHB prespotted spots. Another set of 10 spectra of the $20 \%$ ACN elution fraction of serum glycan is also shown to be highly conserved. 
Miyoung Ha, Youngha In, Hyesun Maeng, Ok Pyo Zee, Jongsik Leea, and Yangsun Kim

(A)

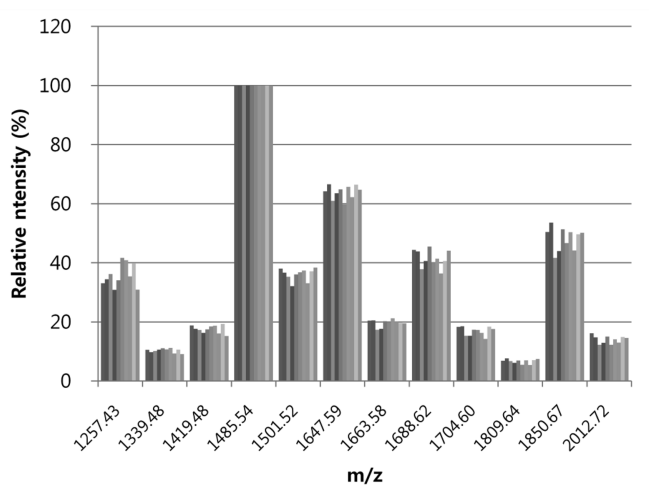

(B)

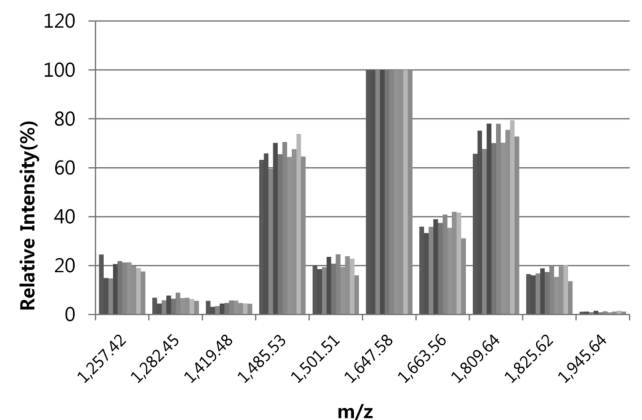

Figure 5. The normalized intensity of significant peaks in (A) $10 \% \mathrm{ACN}$ fraction and (B) $20 \% \mathrm{ACN}$ fraction. The relative distribution of glycans is highly conserved between each spectrum.

Table 1. The averages of relative intensities of significant glycan peaks (Avg), standard deviations (STDEV) and CV (STDEV/Avg $\times$ 100) from 10 different spectra of $10 \%$ ACN elution and $20 \%$ ACN elution each.

\begin{tabular}{ccccccccc}
\hline \hline \multicolumn{4}{c}{$10 \%$ ACN fraction } & \multicolumn{5}{c}{ 20\% ACN fraction } \\
\hline $\mathrm{m} / \mathrm{z}$ & Avg & STDEV CV $(\%)$ & $\mathrm{m} / \mathrm{z}$ & Avg & \multicolumn{1}{c}{ STDEV CV(\%) } \\
1257.43 & 35.73 & 3.90 & 10.92 & 1257.43 & 19.57 & 3.11 & 15.88 \\
1339.48 & 10.32 & 0.72 & 6.97 & 1282.45 & 6.57 & 1.22 & 18.57 \\
1419.48 & 17.35 & 1.34 & 7.66 & 1419.48 & 4.65 & 0.89 & 19.65 \\
1485.54 & 100.0 & 0.00 & 0.00 & 1485.53 & 66.54 & 4.11 & 6.18 \\
1501.52 & 36.09 & 2.06 & 5.71 & 1501.52 & 20.93 & 2.74 & 13.09 \\
1647.59 & 63.90 & 2.29 & 3.59 & 1647.59 & 100.00 & 0.00 & 0.00 \\
1663.58 & 19.65 & 1.22 & 6.22 & 1663.58 & 37.25 & 3.60 & 9.67 \\
1688.62 & 41.47 & 2.97 & 7.17 & 1809.64 & 73.26 & 4.72 & 6.45 \\
1704.60 & 16.86 & 1.50 & 8.89 & 1825.62 & 17.44 & 2.19 & 12.25 \\
1809.64 & 6.68 & 0.76 & 11.44 & 1945.64 & 1.18 & 0.24 & 19.95 \\
1850.67 & 48.21 & 3.86 & 8.00 & & & & \\
2012.72 & 14.01 & 1.32 & 9.43 & & & & \\
\hline
\end{tabular}

\section{Conclusions}

In this paper we developed a homogeneous DHB prespotted MALDI plate which overcomes the large crystal formation of DHB. The reproducible glycan analysis have been presented using MALDI imaging of beer glycan as well as serum glycan eluted from $10 \%$ and $20 \%$ ACN elution fraction. The glycan profile from serum glycan by MALDI-TOF-MS with a DHB prespotted plate was highly conserved for 10 different spectra and the CV of MALDI data is within $10 \%$ for the most abundant ions and less than $20 \%$ for lower abundant ions in the spectra of serum glycan from $10 \% \mathrm{ACN}$ elution and $20 \%$ for ACN elution.

\section{Acknowlegment}

This work was supported by a grant (10035490) of Ministry of Knowledge Economy (MKE), Korea.

\section{References}

1. Wikipedia "Glycosylation", http://en.wikipedia.org/wiki/ glycoprotein.

2. Turnbull, J. E.; Field, R. A. Nat. Chem. Biol. 2007, 3, 74.

3. An, H. J.; Lebrilla, C. B. Mass Spectrom. Rev. 2011, 30, 560.

4. Barkauskas, D. A. I; An, H. J.; Kronewitter, S. R.; de Leoz, M. L.; Chew, H. K.; White, R. W. D.; Leiserowitz, G. S.; Miyamoto, S.; Lebrilla, C. B.; Rocke, D. M. Bioinformatics 2009, 25, 251.

5. Lebrilla, C. B.; An, H. J. Mol. Biosyst. 2009, 5, 17.

6. Kaneshiro, K.; Hukuyama, Y.; Iwamoto, S.; Sekiya, S.; Tanaka, K. Anal. chem. 2011, 83, 3663.

7. Harvey, D. J. Mass Spectrom. Rev. 2011, 30, 1.

8. Wang, H.; Wang, C. H.; Chin, A.; Taguchi, A.; Taylor, A.; Hanashi, S. Nature protocols, 2011, 6, 253.

9. Suzuki, H.; Yamagaki, T.; Tachibana, K. J. Mass Spec. Soc. Japan, 2005, 53, 227.

10. Wilkinson, W. R.; Gusev, A. I.; Proctor, A.; Houalla, M.; Hercules, D. M. Fres. J. Anal. Chem. 1977, 357, 241.

11. Tholey, A.; Heinzle, E. Anal. Bioanal. Chem. 2006, 384, 24.

12. Nicola, A. J.; Gusev, A. I.; Proctor, A.; Jackson, E. K.; Hercules, D. M. Rapid Commun. Mass Spectrom. 1995, 9, 1164.

13. Ha, M.; Maeng, H.; Oh, J.; Kim, Y. $18^{\text {th }}$ International Mass Spectroscopy Conference, Bremen(German), September, 2009.

14. Kronewitter, S. R.; Leoz, M. L. A.; Peacock, K. S.; McBride, K. R.; An, H. J.; Miyamoto, S.; Leiserowitz, G. S.; Lebrilla, C. B. J. Proteome Res. 2010, 9, 4952.

15. Dekker, L. J.; Dalebout, J. C.; Siccama, I.; Jenster, G; Sillevis, P. A.; Smitt, T. M. Rapid Commun. Mass Spectrom. 2005, $19,865$.

16. Gusev, A. I.; Wilkinson, W. R.; Proctor, A.; Hercules, D. M. Anal. Chem. 1995, 67, 1034.

17. Kirmiiz, C.; Li, B.; An, H. J.; Clowers, B. H.; Chew, H. K.; Lam, K. S.; Ferrige, A.; Alecio, R.; Borowsky, A. D.; Sulaimon, S.; Lebrilla, C. B.; Miyamoto, S. Molecular \& Cellular Proteomics 2007, 6, 43.

18. Dekker, L. J.; Burgers, P. C.; Guzel, C.; Luider, T. M. Journal of Chromatogaphy B 2007, 847, 62.

19. Lee, J. H.; Choi, H.S.; Nasr, K. A.; Ha, M.; Kim, Y.; Frangeoni, J. V. Anal. Chem. 2011, 83 (13), 5283. 